\title{
Non-Contact Capacitive Technique for Biomass Flow Sensing
}

\author{
Rumana Tasnim ${ }^{1}$, Sheroz Khan ${ }^{2}$, Atika Arshad ${ }^{3}$, Molla Rashied Hussein ${ }^{4}$ \\ ${ }^{1}$ Department of MTE, World University of Bangladesh, Dhaka, Bangladesh \\ ${ }^{2,3}$ Department of ECE, International Islamic University Malaysia, Kuala Lumpur, Malaysia \\ ${ }^{4}$ Department of CSE, University of Asia Pacific, Dhaka, Bangladesh
}

\begin{tabular}{l} 
Article Info \\
\hline Article history: \\
Received Feb 25, 2018 \\
Revised Apr 7, 2018 \\
Accepted Apr 21, 2018 \\
\hline
\end{tabular}

Keywords:

Biomass

Electrodes

Measurement

Piping

Sensing

\begin{abstract}
To facilitate real-time flow measurement, this paper aims to realize biomass flow sensing through electronic non-contact capacitive means. Hardware implementation has been carried out using a modified OP-AMP-based bridge circuit, with one arm made of a standard capacitance while the other arm is made from two specifically designed capacitive electrodes fitted on a piping system sensing biomass flow. The experimental results are targeted to obtain data for given biomass types through a custom-developed biomass flow piping system. Several flow affecting parameters namely: electrodes' shapes, the location of electrodes on the piping system, biomass material type, and particle size have been considered in obtaining experimental data. Also, the circuit has been simulated to analyze flow sensing behavior for the proposed technique by evaluating the measurement data and assessing conformity between experimentally obtained and simulated data.
\end{abstract}

Copyright (c) 2018 Institute of Advanced Engineering and Science. All rights reserved.

\section{Corresponding Author:}

Rumana Tasnim,

Department of MTE,

World University of Bangladesh, Dhaka, Bangladesh.

Email: rumanatasnim415@gmail.com

\section{INTRODUCTION}

On-line line mass flow sensing and measurement of particulate biomass solids on a pneumatic pipeline has an imperative role to improve process efficiency. Currently, the use of biomass fuels has become extensive in an effort to substitute manual supply of coal by a continuous and measured coal supply at power stations. Moreover, biomass is solely imperative for energy and materials conversion processes given the relative abundance of biomass waste materials, the renewable nature of biomass and its favorable status regarding emission of greenhouse gases. Generally speaking, biomass solids can appear in the form of powder and grain transported in gases (typically air) which has widespread applications across industries of numerous types including food processing, agricultural particles (e.g., grains, wheat, corn) [1] flowing, pharmaceutical and petrochemical, cement, mining, semiconductor, chemical and also in transporting pulverized coal in fuel lines of thermal power plants. Recently a novel electrostatic sensing technique has been developed to conduct the online continuous measurement of "mean" and "local" characteristics of blended biomass flow on pneumatic driven pipelines at coal power station using circular and arc-shaped electrostatic sensor arrays in order [2].

Some other researchers worked on designing capacitive sensor front-end emphasizing the range of measurement sensitivity of the setup [4-5], robustness to electromagnetic interference along with the assessment of shielding techniques. Some researchers have come forward with innovative electrode designs and tested those in versatile hardware platform which allows reliable testing of the sensor structure, capacitance measurement circuit and topologies in different applications devoid of improving data acquisition, communication and implementation. For making capacitance-to-digital (CDC) conversion, few capacitance measurement schemes are implemented in the form of integrated Circuit (IC) [6]-[7]. In [8] the authors experimented on an off-line gas-solids flow instrumentation method using a 150mm bore commercial 
capacitance transducer (DC 13) and the solids concentration meter (DMC 170). The measured capacitance is converted into voltage signal through integrating capacitor in an electronic circuit which is rectified, amplified, and onward transmitted to DMC 170 in the form of pulse frequency.

In [9], the authors developed semi cylindrical capacitive sensor with an interface circuit for flow rate measurement. The measurement results were moderately satisfactorily which proved the performance of the semi cylindrical capacitive sensor with an interface circuit for flow rate measurement. The aforementioned research studies in the area of flow measuring sensors have been becoming a source of rehabilitated interest among researchers these days. The issues concerning the reported capacitive sensing measurement technologies are electrode structure designing and optimization, pipeline design, complicated and unreliable capacitance measurement circuits, effects of unusual particle properties on sensing phenomenon for pneumatic conveying of biomass. This paper aims to carry out experimental and simulation analysis on biomass particle flow sensing by using semi-circular (e.g., SA. SB, SC) and circular (e.g., CA, CB, CC) shaped capacitive sensor electrodes with a cost effective measurement circuit which is capable of perceiving biomass flow patterns and eliminating stray capacitance to a large degree.

\section{CAPACITIVE SENSING}

Capacitive sensing allows the conversion of biomass flow variation into capacitance value. The capacitive sensor is constructed with a pair of sensing electrodes. An interface circuit which is also referred as a measurement circuit is employed for representing the capacitance value into ac voltage signal. The block diagram of the whole measurement and sensing system is as shown in Figure 1.

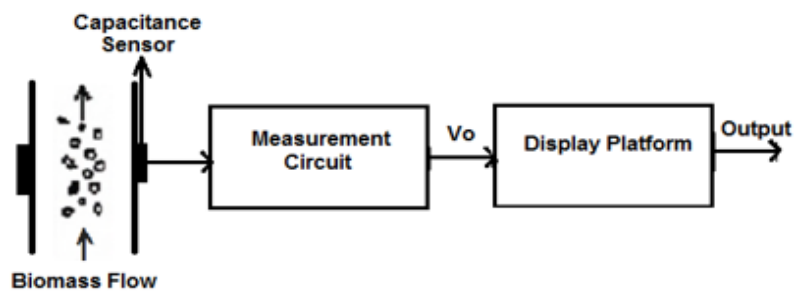

Figure 1. Block diagram of a capacitive sensing technique

\section{PROBLEM STATEMENT LEADING TO OBJECTIVE}

The significance and magnitude of measuring an accurate flow rate of gas-solid flow in pipelines has been ever-increasing in the industrialized regions. From literature review, few issues have been highlighted on the way to conduct our research.

1) Not much research has been undertaken on the perception of flow pattern and sensing behavior in term of the signal detected which influences the measurement procedure of concentration and velocity of biomass particles.

2) Due to the technique of handling irregular biomass particles and complicated physical characteristics, the transportation of solid biomass by air is typically unpredictable. This leads toward limited industrial biomass processes; so the potential of biomass as a source of renewable energy is greatly unrealized.

3) In spite of the wide-ranging significance of this renewable energy source, biomass multiphase flows have achieved very limited attention. Consequently, the prospective of industrial biomass process instrumentation is also not perceived. Further research work should be called for enhancing understanding of the multiphase biomass pneumatic conveying system and realizing the multiphase flow of biomass materials with the purpose of improving biomass process instrumentation and also utilizing biomass resources effectively in this complicated area of research.

The reported capacitive sensing measurement technology used for measuring gas/solid particles' velocity and concentration lack a good level of efficiency since some measurement metrics appears to affect expected outputs. Ultimately the flawed measurement procedure results in poor performance of the overall system. Most of the recent research and studies have been concerned with the circular and rectangular shaped pipe using circular, arc, planner, multi-electrode, pin-shaped electrodes for gas-solid particle flow measurement namely coal. No research has focused on biomass particle flow sensing by the semi-circular shaped capacitive sensor with cost effective measurement circuit that involves the proper perception of biomass flow pattern. Moreover, measurement problem regarding the measurement of very small 
capacitances is mentionable since cable capacitance and stray capacitance cause inaccuracy. Also, power line interference and interface circuit drift are other issues of concern for research in this work. The problems highlighted to resolve for this research work are summarized below:

1) Complexity in existing measurement circuit model as well as electrode arrangement used for flow piping arrangement.

2) Effect of stray capacitance and residual capacitance effect in measurement circuit.

3) Not much research has been undertaken to analyze sensing behavior of biomass flow.

For facilitating the sensing of small capacitive variation and solving the above mentioned problems, a capacitive sensing techniques has been developed for monitoring and analyzing the sensing behaviors of biomass flow through reducing stray capacitance effect. This research enables the biomass flow sensing using an operational amplifier based capacitive bridge circuit, reproducing the output voltage as a representative of the flow.

\section{METHODOLOGY}

With the aim of measurement for real-time monitoring in mind, a low cost capacitance sensing OPAMP-based circuit derived from [3] for blended biomass flow is developed. The biomass flow sensing system structure (as shown in Figure 2) consists of two parts: (a) sensing part that includes piping system which has biomass container at one end, electrodes fitted on the vertical up, horizontal and vertical down sections of the piping systems through which biomass is pneumatically sucked by a suction pump fitted at the far end; (b) processing circuit which is analysed in the form of deriving its mathematical equations relating the output voltage to capacitance changes.

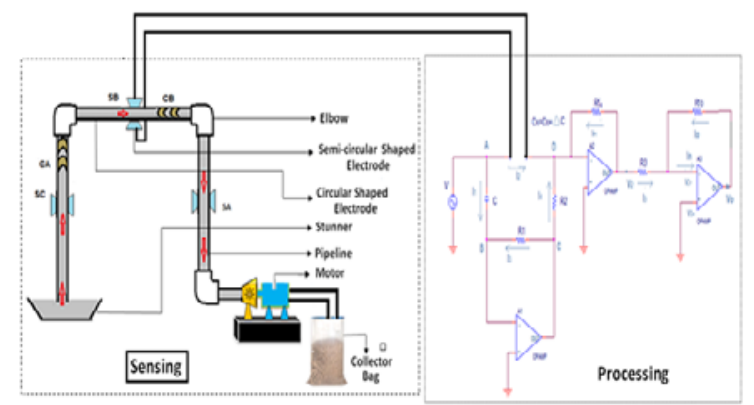

(a)

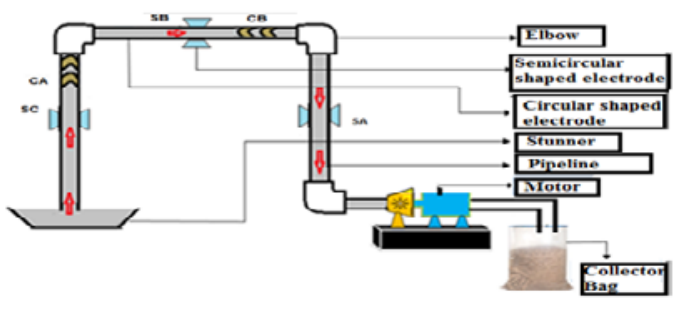

(b)

Figure 2. (a) System structure with sensing and processing part, (b) Sensor arrangement

The two processing parts are explained as follows:

a) Sensing Part

The test pipe section (as in Figure 2(b)) made of polyvinyl chlorides constructed with vertical and horizontally connected pipe of length $1000 \mathrm{~mm}$ with an upward flow direction. The pipe has inside diameter of $25 \mathrm{~mm}$ and outside diameter of $27 \mathrm{~mm}$. In the test section, five capacitance sensor electrodes for the measurement of capacitance are located keeping certain distances throughout the piping system.

On the test pipe, a low cost solely noncontact type circular electrodes (made of mild steel) and semicircular (made of galvanized steel) shaped capacitive sensor electrodes have been placed to make up one arm of the bridge circuit. The design parameters for the electrodes have been chosen such that the ratio of electrode width (W) to electrode separation (d)remains at 4:1, the number of electrodes, $K=10$, the electrode width of circular and semi-circular electrode is kept at $12 \mathrm{~mm}$, electrode separation is $\mathrm{d}=3 \mathrm{~mm}$, radius of the pipe, $r=12.5 \mathrm{~mm}$. Test material ground grain corn flour (shaped as $100 \mu \mathrm{m}$ ) is replicated as fine biomass flow base [2] and biomass fuel wood $(200 \mu \mathrm{m}-2 \mathrm{~mm})$ is made bigger than that of corn flour.

b) Processing Part

The test pipe section (as in Figure 2(b)) made of polyvinyl chlorides constructed with vertical and horizontally connected pipe of length $1000 \mathrm{~mm}$ with an upward flow direction. The pipe has inside diameter of $25 \mathrm{~mm}$ and outside diameter of $27 \mathrm{~mm}$. In the test section, five capacitance sensor electrodes for the measurement of capacitance are located keeping certain distances throughout the piping system.

The flow pipe is inserted as a lone arm of the bridge, while other arms with given values of resistive and capacitive elements. The four arms of the bridge are used in two pairs: the upper pair is made of capacitances, one fixed while another is representing variable capacitor with values representing equivalent biomass flow. The lower pair of bridge arms is made of resistors only while keeping point B at a virtual 
ground for better stability of flow sensing by the variable capacitance arm, Cx (as in Figure 2). The last OPAMP is used as a buffer, stopping the load from affecting the measurement made; it makes an effort to make such measurements true representative of biomass flow. The second (the middle) OP-AMP is used for providing adjustable sensitivity to the overall results. In this circuit same potentials has been maintained at the output nodal points of the bridge circuit. Table 1 illustrates circuit components' specification. From the circuit, the bridge output is the voltage which is linearly related with the minute changes in the capacitance $\Delta \mathrm{C}$ of the sensor, and hence, it will be linearly related with the concentration of the biomass flow. The voltage output equation derived for the circuit is:

$$
V_{0}=\frac{j \omega V R_{f_{0}} R_{f 1}}{R_{3} R_{2}}\left(C_{x}-C_{0}\right)
$$

Table 1. Specification of circuit components

\begin{tabular}{ccccccccc}
\hline $\mathrm{C}$ & $\mathrm{C}_{0}$ & $\mathrm{R}_{1}$ & $\mathrm{R}_{2}$ & $\mathrm{R}_{3}$ & $\mathrm{R}_{\mathrm{f} 1}$ & $\mathrm{R}_{\mathrm{f} 2}$ & $\mathrm{C}_{\mathrm{R} 1}=\mathrm{C}_{\mathrm{R} 2}$ & $\mathrm{C}_{\mathrm{R}} \neq \mathrm{C}_{\mathrm{R} 2}$ \\
\hline $10 \mathrm{pF}$ & $10 \mathrm{pF}$ & $1 \mathrm{k}$ & $1 \mathrm{k}$ & 10 & $100 \mathrm{k}$ & $100 \mathrm{k}$ & Balanced (no flow) & Unbalanced (with flow)
\end{tabular}

\section{RESULTS AND ANALYSIS}

Experimentally the bridge circuit is made balanced under no flow conditions; only to become unbalanced with biomass flow. The circuit is tested by allowing wood biomass at 333g/min and corn flour at $600 \mathrm{~g} / \mathrm{min}$. The flowing speed of materials differs from each other depending upon the biomass material in use. The capacitance changes for each material are recorded every 10 seconds over a period of 60 seconds. The experimental results are plotted for wood flow in Figure 3 (a)-(b), illustrating the plots of output voltage and capacitance variation in proportion to the flow being sensed.

Figure 3 (a) and (b) illustrate the output voltage and capacitance plots for circular and semi-circular electrodes. The circular electrodes (CA) and semicircular electrode (SC) are fitted on upward flow pipe, the vertical section near to the container. Circular electrode (CB) and semicircular electrode (SB) are mounted on the horizontal section, the middle segment of the piping system. Another semicircular electrode (SA) is placed on downward flow pipe, the vertical pipe section near to the suction pump. The observations made are:

1. The output voltages measured at circular electrodes has shown identical patterns as a measure of capacitance changes and the semicircular ones show approximately similar behavior in their output voltages indicating the authenticity of the measurement made.

2. Semicircular SC and circular CA fitted near the container have shown maximum output voltage. Due to the location of SC and CA electrode near the biomass container, solid concentration has displayed higher capacitances than that of other pipe sections, and that indication in the form of the higher output voltage is proved accordingly.

3. However, two horizontally positioned electrodes (CB) and (SB) in the middle pipe have demonstrated less-fluctuations in capacitance values due to location reason. The semicircular electrode SA has shown almost stable but smaller fluctuation in capacitance plot due to the less concentration in this section during the entire flow.

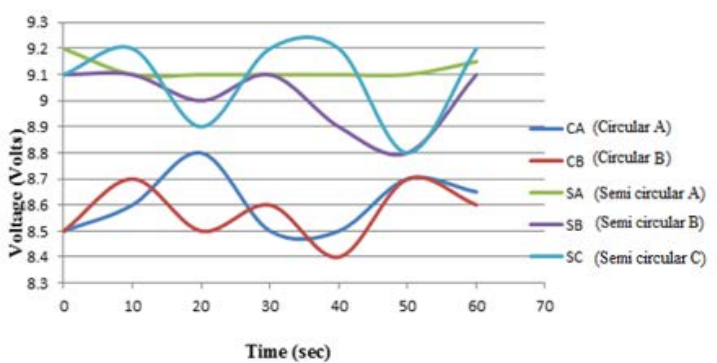

(a)

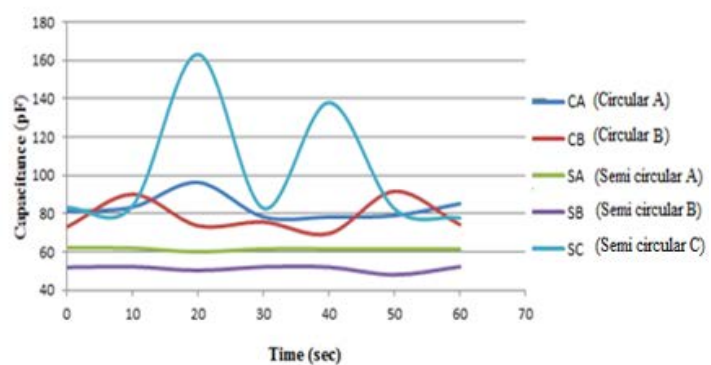

(b)

Figure 3. (a) Voltage Output as a function of wood flow (333grams) over 60 seconds, (b) Capacitance as a function of wood flow (333grams) over 60 seconds 
The bar graphs of Figure 4 (a-e) for different electrodes have proven that the sensing capacitance directly affects the output voltage obtained. The test is repeated again with the same flow regime for corn biomass flow (600g)where the diameter of its' particles size is $50 \mu \mathrm{m}$ and it is flown over 60 seconds of the period that move faster than the wood biomass due to the fine particle size characteristics of the material. Unlike wood biomass, the flow rate is relatively higher in case of corn particles; so the concentration of these moving materials is more than that of wood biomass over the same time span.

Figure 5(a) and (b) illustrate the output voltage and capacitance plot as a function of corn flour flow over 60 seconds respectively.

1) Two electrodes (CB and SB) fitted on the horizontal piping system have shown the lowest capacitance values while the electrodes placed near biomass container (CA and SC) have shown the maximum capacitance values which is in good agreement with the results reported for wood biomass. The capacitances measured at those points have shown maximum capacitance values due to the higher solid concentration values. These results are identical to voltage measurement plots. The output voltages measured at CA and CB have shown similar variation pattern in form of capacitance measured.

2) The only semicircular capacitance electrode SA mounted near the suction pump has revealed a stable line in capacitance graph due to its location. The concentration on that sub section area remains almost same during the entire period of wood flow.

3) Among the circular electrodes, CA has shown relatively bigger capacitance sensing; that is due to its' location nearer to biomass container. Among the semicircular ones, SB has shown lower capacitances due to once again location reason on horizontal sections. The horizontally flown biomass materials do not scatter and spread over the sensing volume unlike vertically flown biomass. The pressure drop is due to two bents on the piping system, showing a stable concentration on the horizontal pipe, maximum near the container and low near the suction pump.

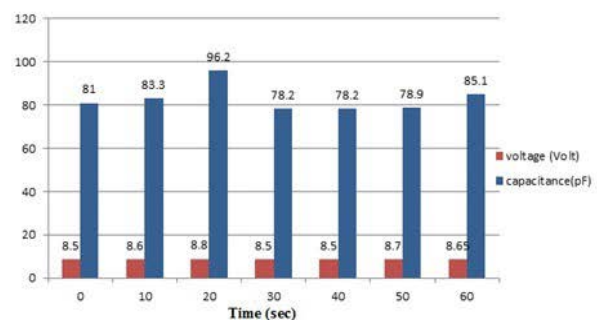

(a)

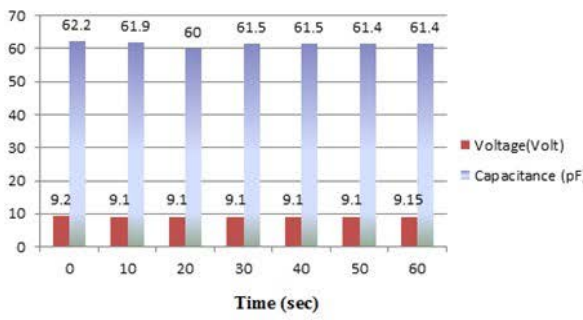

(c)

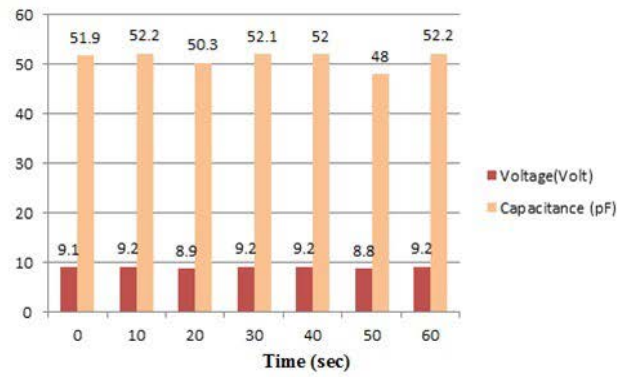

(e)

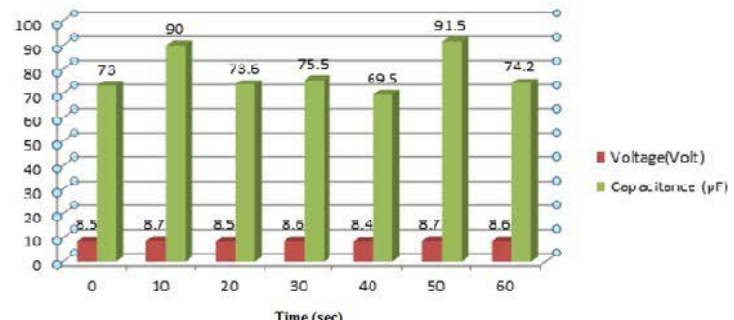

(b)

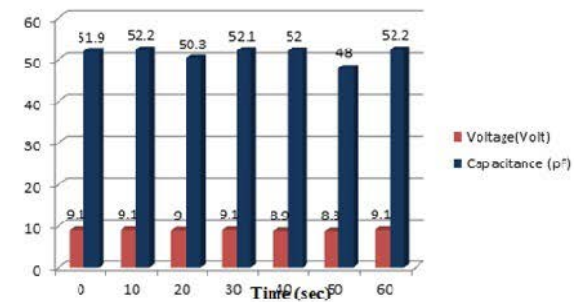

(d)

Figure 4. Bar graph of wood flow over one minute (333gm/min) (a) Sensed by CA (b) Sensed by CB (c) Sensed by SA (d) Sensed by SB(e) Sensed by SC Capacitance as a function of wood flow (333grams) over 60 seconds 


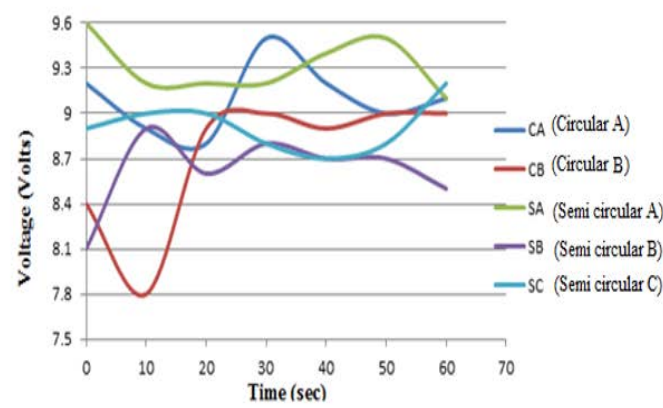

(a)

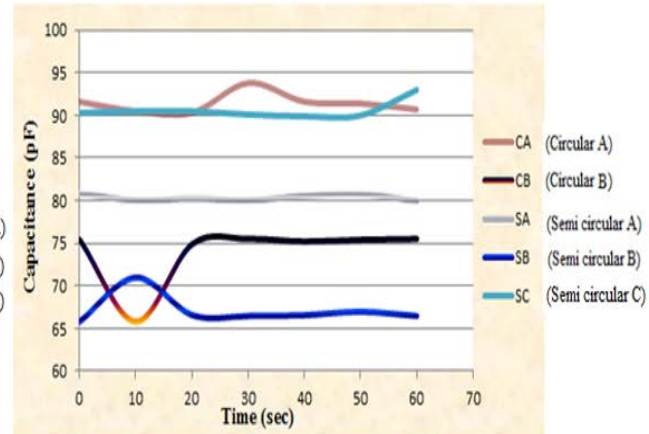

(b)

Figure 5. (a) Voltage Output as a function of corn flow (600gm/min), (b) Capacitance as a function of corn flow $(600 \mathrm{gm} / \mathrm{min})$

The peaks and dips of capacitance graphs in Figure 5(b) indicate that the circular electrodes sensed the concentration of corn flow materials giving a steady response. A similar set of bar graphs are found also for corn flour [as in Figure 6(a-e)].

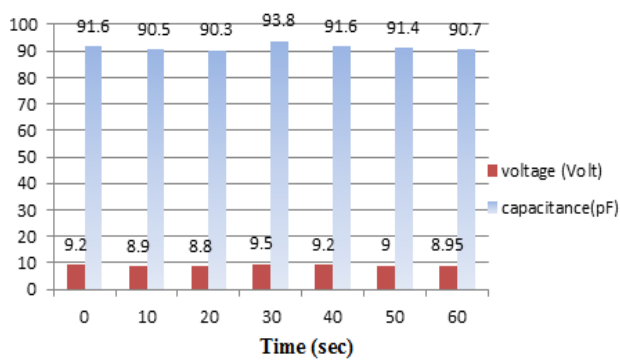

(a)

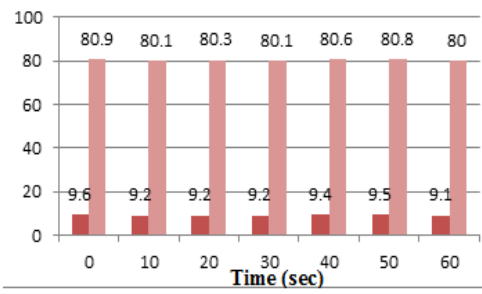

(c)

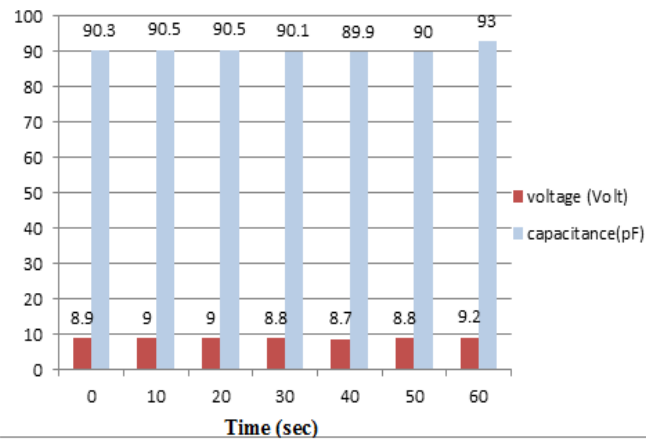

(e)

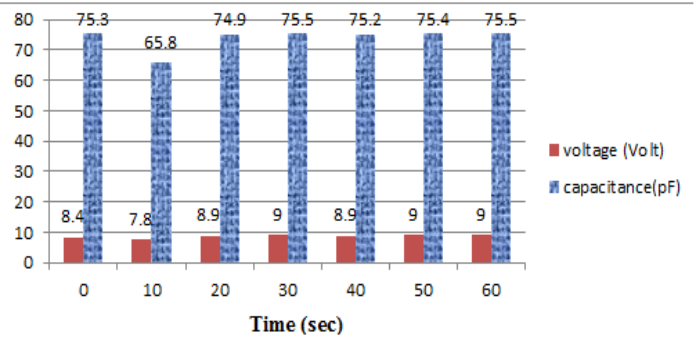

(b)

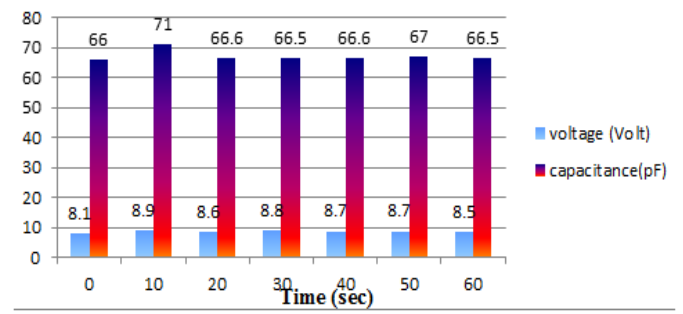

(d)

Figure 6. Bar graph of corn flour flow $(600 \mathrm{gm} / \mathrm{min})$ for (a) circular electrode CA (b) circular electrode CB (c) semicircular electrode SA (d) semicircular electrode SB (e) Semi-circular electrode SC 
Moreover, simulation has been carried out to obtain output voltage for the obtained capacitances sensed by the sensing electrodes and compared with the experimentally achieved output voltages Vo as a function of different capacitance changes in Cx. These experimental results are ascended in Table 2 which is based upon the wood flow of $(333 \mathrm{gm} / \mathrm{min})$ for circular electrode CA. Moreover, PSpice simulation results with equivalent capacitance $\mathrm{Cx}=\mathrm{CA}=83.3 \mathrm{pF}$ is shown Figure 7.

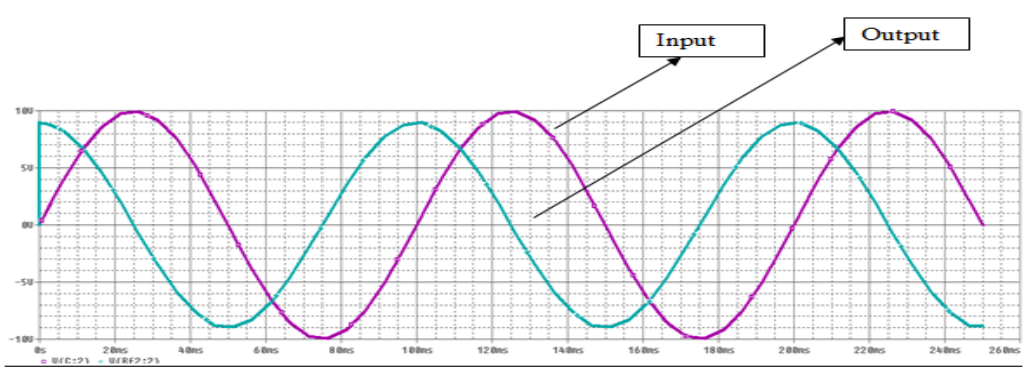

Figure 7. PSpice Simulation results for $\mathrm{Cx}=\mathrm{CA}=83.3 \mathrm{pF}$

Figure 7 shows a specific voltage (8.9 volts) obtained from simulation result for a distinctive value of experimentally sensed capacitance across CA (Cx) electrode. A set of simulated voltages are found for the capacitances ranging from 78.2 to 96.2 (as in Table 2). A similar relationship is observed (as in Table 3) for the same wood flow sensed by semi-circular SA over 60 seconds. Simulation is carried out using PSpice for the capacitances varying from 60 to $62.2 \mathrm{pF}$.

Table 2. Experimental and simulated output voltages arranged in ascended manner for wood flow sensed by CA

\begin{tabular}{ccc}
\hline Capacitance $(\mathrm{pF})$ & Voltage in Volts (Experimental) & Voltage in Volts (Simulation) \\
\hline 78.2 & 8.5 & 8.3 \\
78.2 & 8.5 & 8.3 \\
81 & 8.5 & 8.5 \\
83.3 & 8.6 & 8.9 \\
85.1 & 8.65 & 9.1 \\
96.2 & 8.8 & 10.1 \\
\hline
\end{tabular}

Table 3. Experimental and simulated output voltages arranged in ascended manner for wood flow sensed by SA

\begin{tabular}{ccc}
\hline Capacitance $(\mathrm{pF})$ & Voltage in Volts (Experimental) & Voltage in Volts (Simulation) \\
\hline 60 & 9.1 & 6.2 \\
61.4 & 9.1 & 6.4 \\
61.4 & 9.15 & 6.4 \\
61.5 & 9.1 & 6.4 \\
61.5 & 9.1 & 6.4 \\
62.2 & 9.2 & 6.6 \\
\hline
\end{tabular}
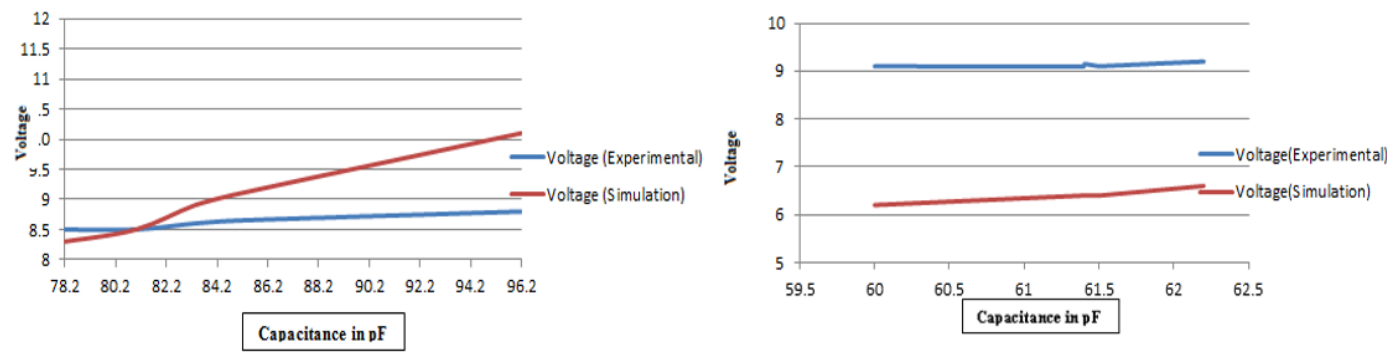

Figure 8. (a) Experimental and Simulation Voltage for wood flow sensed by CA, (b) Experimental and Simulation Voltage for wood flow sensed by SA 
Figure 8(a) illustrates that simulation and experimental results linearly vary in the larger part of capacitance sensing. A similar relationship has been observed for the same amount of wood biomass flow (333gm/min) sensed by the semi-circular electrode SA over 60 seconds of time. Figure $8 \mathrm{~b})$ illustrates simulation and experimental output voltages which maintained similar linear variation in output voltages. In case of circular electrodes, the difference in the voltage output is not observed to be as large as semicircular one. Also, circular electrodes showed better conformity but least sensitivity between the simulated and experimented output voltages, unlike semicircular ones. Capacitance values sensed for fine corn flour, once again the simulation results are obtained.

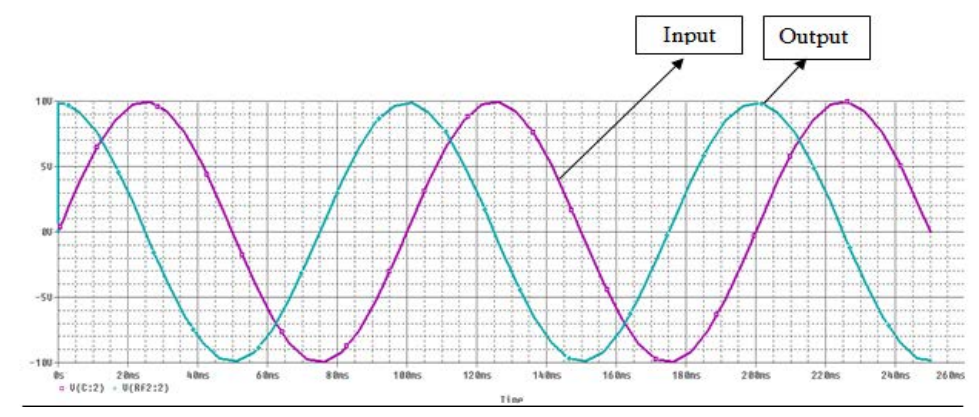

Figure 9. PSpice simulation result for $\mathrm{Cx}=91.6 \mathrm{pF}$

Figure 9 suggests a specific voltage of 9.8 volts from simulation result for a distinctive capacitance value of $91.6 \mathrm{pF}$. Accordingly, a set of simulated voltages are recorded for capacitances ranging from 90.3 to $93.8 \mathrm{pF}$ as shown in Table 4. This tabulated data for experimental and simulation and experimental results based on non-uniform corn flour flow $(600 \mathrm{~g} / \mathrm{min})$ using circular electrode CA is as shown in Figure 10.

Table 4. Experimental and simulated output voltages arranged in ascended manner (for corn flour flow

\begin{tabular}{ccc}
\multicolumn{3}{c}{ sensed by CA) } \\
\hline Capacitance(pF) & Voltage in Volts (Experimental) & Voltage in Volts (Simulation) \\
\hline 90.3 & 8.8 & 9.6 \\
90.5 & 8.9 & 9.6 \\
90.7 & 8.95 & 9.7 \\
91.4 & 9 & 9.7 \\
91.6 & 9.2 & 9.8 \\
93.8 & 9.5 & 10.1 \\
\hline
\end{tabular}

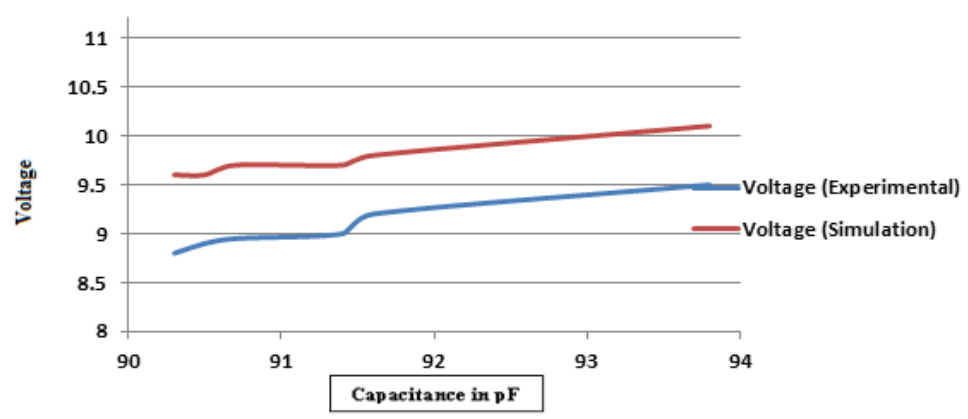

Figure 10. Experimental and Simulation Voltage for corn flour flow across CA

Figure 10 illustrates a similar pattern of simulated and experimental results obtained with similar variation slope in output voltages, once again the simulation data showed better sensitivity in case of circular electrodes. As a continuation of the previously reported result, simulation results obtained for capacitance sensed by SA and experimental results are noted in Table 5, the results are plotted in Figure11. 
Table 5. Experimental and simulated output voltages arranged in ascended manner (for

\begin{tabular}{ccc}
\multicolumn{3}{c}{ corn flour flow sensed by SA) } \\
\hline Capacitance(pF) & Voltage in Volts (Experimental) & Voltage in Volts (Simulation) \\
\hline 80 & 9.1 & 8.5 \\
80.1 & 9.2 & 8.5 \\
80.1 & 9.2 & 8.5 \\
80.3 & 9.2 & 8.6 \\
80.6 & 9.4 & 8.7 \\
80.8 & 9.5 & 8.8 \\
\hline
\end{tabular}

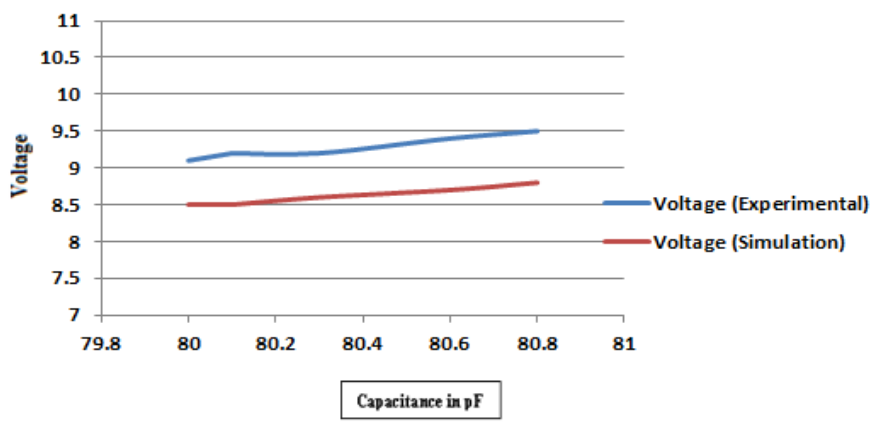

Figure 11. Experimental and Simulation Voltage for corn flour flow across SA

In term of consistency between experimental and simulation results, Figure 11 has shown better consistency for semi-circular electrodes than that of circular electrodes.

The capacitive measurement of biomass concentration obtains solid phase concentration by measuring the capacitance of capacitive sensor based on the established relationship between solid phase concentration and measurement capacitance (Cx).

$$
\rho=\rho_{s}\left(C_{x}-k^{\prime} \in_{g}\right) / k^{\prime}\left(\epsilon_{s}-\epsilon_{g}\right)
$$

Where, $\rho=$ Biomass (solid phase) concentration,

$\rho_{s}=$ Biomass (solid phase) density

$\epsilon_{s}=$ Biomass (solid phase) permittivity

$\epsilon_{g}=$ Air (gas phase) permittivity

$k^{\prime}=$ addressed by structure and dimension of capacitance sensor plates

$\left(\mathrm{C}_{\mathrm{x}}\right)=$ Measurement capacitance $\left(\mathrm{C}_{\mathrm{x}}\right)$

From Equation (1),

$$
\begin{aligned}
& V_{o} R_{3} R_{2}=-j \omega v R_{f o} R_{f 1}\left(C_{x}-C_{0}\right) \\
& \Rightarrow j \omega v R_{f o} R_{f 1} C_{x}=j \omega v R_{f o} R_{f 1} C_{0}-V_{o} R_{3} R_{2} \\
& \Rightarrow C_{x}=\frac{j \omega v R_{f o} R_{f 1} C_{0}-V_{o} R_{3} R_{2}}{j \omega v R_{f o} R_{f 1}}
\end{aligned}
$$

And from Equation 2,

$$
\begin{aligned}
& \rho=\rho_{\mathrm{s}}\left(\mathrm{C}_{\mathrm{x}}-\mathrm{k}^{\prime} \epsilon_{\mathrm{g}}\right) / \mathrm{k}^{\prime}\left(\epsilon_{\mathrm{s}}-\epsilon_{\mathrm{g}}\right) \\
& \Rightarrow \rho=\frac{\rho_{\mathrm{s}}\left\{\mathrm{C}_{0}-\frac{\mathrm{v}_{\mathrm{o}} \mathrm{R}_{3} \mathrm{R}_{2}}{\mathrm{j} \mathrm{vR}_{\mathrm{fo}} \mathrm{R}_{\mathrm{f} 1}}\right\}-\mathrm{k}^{\prime} \epsilon_{\mathrm{g}}}{\mathrm{k}^{\prime}\left(\epsilon_{s}-\epsilon_{\mathrm{g}}\right)}
\end{aligned}
$$

So, biomass concentration is derived with respect to measured capacitance and voltage output in equation 3. This has established a correspondence relationship between biomass concentrations with biomass and air phase density, dielectric permittivity of test biomass materials as well the physical characteristics and structure of the capacitive electrode. The experimental results have shown likewise behaviour. 


\section{DISCUSSION}

To realize the sensing behavior of different biomass test materials this work has been carried out by tuning the findings to achieve the research objectives. The developed piping system, electrodes and its circuit is capable of detecting very small capacitance variations while minimizing or offsetting the effect of stray and residual capacitances during flow. The overall results shown prove the validity of the circuit to be used in real time flow measurement scheme when connected to a programmable data processing unit in a Programmable Logic Controller (PLC)-based environment.

The section close to the biomass container showed larger concentration values (both in terms of capacitance and voltage sensed) than the remaining parts of the system. The section close to the suction pump show steady but less values of output voltage and capacitance changes. The physical properties of fine particle corn are comparatively different from wood that influences the measurement made as better steady output.

Since the galvanized iron semi-circular electrodes have better conductivity than that of mild steel circular ones, sensitivity have been proven to be better in case of semicircular ones. Some valid comparisons have been made between the capacitance sensed and voltage values in term of simulation and experiments. The experimental and simulated voltages suggest that the semi-circular electrodes show better sensitivity in case of the biomass flow while maintaining a general consistency over the given range of measurement. Simulation and experimental consistency has been proven to be relatively more linear over small capacitive variation for semicircular electrode rather than circular ones.

\section{CONCLUSION}

In order to carry out flow sensing and measurement of biomass in an industrial environment, the results of this research work has proven the utility by setting up a prototype experimental setup. For real-time flow measurement, proposed sensing technique can be connected with data processing unit from where the plant operators can provide useful data to enhance flow control system performance. The results have shown distinctive features based on shapes and physical characteristics of the electrodes in use, locations of the mounted electrodes on test pipe and dielectric permittivity of biomass materials. Results from the tests conducted agree that the approach with measurement circuit and dissimilar electrodes have worked out very well with the detection of flow variation in very small capacitance changes. Further, the two types have shown consistent results between simulation and experimental validation. For further research progress, the whole system needs to be tested using a properly developed measurement bed, connected to a data processing unit in the SCADA-based industrial environment.

\section{ACKNOWLEDGMENT}

Financial assistance for this research by the IIUM Research Management Center (RMC) via RIGS Grant No RIGS 15-147-0147 is highly acknowledged.

\section{REFERENCES}

[1] Heping Cui \& John R. Grace (2006) Pneumatic conveying of biomass particles: a review, China Particuology Vol. 4, Nos. 3-4, 183-188.

[2] Xiangchen Qian \& Yong Yan. (2012, May). Flow Measurement of Biomass and Blended Biomass Fuels in Pneumatic Conveying Pipelines Using Electrostatic Sensor-Arrays. IEEE Transactions on Instrumentation and Measurement, 61 (5), pp. 1343- 1352.

[3] Bera, S. C., \& Mandal, H., (2012, September). A Flow Measurement Technique Using a Noncontact CapacitanceType Orifice Transducer for a Conducting Liquid. IEEE Transactions on Instrumentation and Measurement, vol. 61, NO. 9, 2553

[4] Lucas, J., Hole, S., \&Batis, C. (2006). Analytical Capacitive Sensor Sensitivity Distribution and Application. Measurement Science and Technology, 17, pp. 2467-2478.

[5] Zangl, H., Bretterklieber, T., \& Holler, G. (2008). On the Design of a Fully Differential Capacitive Sensor FrontEnd Interface Circuit. Microelectronics Conference ME. 2008, pp. 132

[6] Bretterklieber, T., Zangl, H., Hrach, D., Holler, G., Hammerschmidt, D., \&Motz, M. (2008).Versatile Programmable Integrated Interface for Robust Capacitive Sensors. Elektrotechnik and Informationstechnik, 125 (4), pp.123-128.

[7] Bretterklieber, T., Zangl, H., Motz, M. \&Werth, T. (2008).Versatile Sensor Front End for Low- Depth Modulation Capacitive Sensors. IEEE International Instrumentation and Measurement Technology Conference, pp. 830-835.

[8] Yan, Y., \& Reed, A.R. (1999). Experimental evaluation of capacitance transducers for volumetric concentration measurement of particulate solids. Flow Measurement and Instrumentation, pp.45-49. 
[9] Cheng-Ta Chiang \& Yu-Chung Huang. (2006, December). A Semi cylindrical Capacitive Sensor with Interface Circuit Used for Flow Rate Measurement. IEEE Sensors Journal, 6 (6), pp. 1564-1570

[10] T.S. Gunawan, I.R.H. Yaldi, M. Kartiwi and H. Mansor, "Performance Evaluation of Smart Home System using Internet of Things," International Journal of Electrical and Computer Engineering (IJECE), 8(1), pp.400-411, 2018.

[11] D Istardi, A Triwinarko. Induction Heating Process Design Using COMSOL ${ }^{\circledR}$ Multiphysics Software. TELKOMNIKA (Telecommunication, Computing, Electronics and Control). 2011; 9(2): 327-334. 